\title{
Cálculo de caudales de mantenimiento en ríos de la cuenca del Tajo a partir de variables climáticas y de sus cuencas
}

\author{
Domingo Baeza Sanz \& Diego García de Jalón. \\ Laboratorio de Hidrobiología, E.T.S. de Ingenieros de Montes. Universidad Politécnica de Madrid. Avda. \\ Ramiro de Maeztu s/n 28040 Madrid. España
}

\begin{abstract}
RESUMEN
Dentro de las metodologías para el cálculo de un caudal de mantenimiento o ecológico para nuestros ríos, hemos estado utilizando un análisis de caudales históricos. Hemos encontrado un caudal mínimo que, a diferencia de los obtenidos en otros métodos, donde ese caudal representa un determinado valor que ha circulado puntualmente en el río y de forma excepcional, se pretende obtener un caudal bajo que ha estado mantenido en el río durante un periodo largo de días, alrededor de 25 . Esta metodología pensamos que es más válida pues tiene mayor sentido biológico, ya que supone unas condiciones perturbadoras continuadas a las que se han tenido que adaptar las poblaciones del río. En este trabajo intentamos relacionar este caudal con variables climáticas, hidrológicas y de la cuenca. También se ha intentado utilizar las clasificaciones de estos ríos, que se han obtenido en estudios anteriores, en los que hemos utilizado este parámetro y otros que nos caractericen el régimen en cuanto a predicibilidad, constancia y estacionalidad de caudales. Dentro de las relaciones encontradas se ha observado que todos los caudales de mantenimiento de todos los ríos presentaban una alta correlación con el caudal medio y la superficie de la cuenca; algunos grupos también se veían influidos por la ETP, y factores erosivos de los utilizados en el método USLE, en los que los valores se tomaron del mapa de estados erosivos de la cuenca del Tajo, correspondientes a las cuencas estudiadas.
\end{abstract}

Palabras clave: Cuenca hidrográfica, caudal ecológico, correlación, módelos matemáticos.

\begin{abstract}
We have analysed historical flows to estimate basic or ecological flows for our rivers. We have found that the minimum flow, unlike values of flows obtained using other methods where flow represents an individual and exceptional value, is better indicator of flows which have been maintained for a long period (i.e. around 25 days). We believe that this methodology is more valid and results are easier to interpret in biological terms. The method takes into account prolonged perturbing conditions to which river populations have had to adapt. In this paper, we try to relate this flow with climate, hydrological and basin variables. Also, we use previous classifications for these rivers as a guide to predictability, constancy and seasonality of flows. From this comparison we observed that basic flows of all rivers were highly correlated with average flows and basin area. In addition, some groups were seen to be influenced by ETP, area forested and erosion levels in the study basin, on the basis of factors integrating the U.S.L.E. model of erosive states in the Tagus basin.
\end{abstract}

Keywords: Hydrographic basin, ecological flow, correlation, mathematical models.

\section{INTRODUCCIÓN}

Las actuaciones hidráulicas realizadas muy intensamente en las últimas décadas en nuestro país han alterado, de forma muy notable, el régimen de caudales de nuestros ríos, así como el funcio- namiento de los ecosistemas acuáticos, y en algunos casos las características de la cuenca.

Para tratar de restablecer un régimen de caudales que mantenga el ecosistema dentro de unos valores de funcionamiento, similares a los de las condiciones naturales, ha aumentado el interés

Limnetica 16: 69-84 (1999)

(C) Asociación Española de Limnología, Madrid. Spain. ISSN: 0213-8409. 
por los estudios del régimen de caudales naturales y de las variables que los condicionan. Debido a que la situación normal es la de sustraer caudales al río, se presta especial atención a las situaciones de caudales mínimos.

Las variables de la cuenca que controlan el régimen de caudales pueden ser utilizadas para establecer modelos, de los que se obtienen valores que pueden servir como estimadores de las caudales mínimos o de mantenimiento, y así responder a las peticiones que de estos caudales requieren los organismos de cuenca.

Hay pocos trabajos en los que se estudia las modificaciones que parámetros de cuenca pueden producir en los caudales de los ríos. Se han encontrado relaciones entre el caudal, la escorrentía y la precipitación y densidad de drenaje (longitud de los cauces por unidad de área), con respecto a variables geomorfológicas, características de la cuenca y clima (Morisawa 1962, 1968; Nelson et al. 1992).

También en la hidrología torrencial se han relacionado las variables utilizadas en hidrogramas geomorfológicos y con los caudales de avenida, que se producen en días de lluvias torrenciales (Valdes et al., 1979).

En ríos australianos, Mc Mahon (1982) ha elaborado un método para calcular caudales anuales a partir de variables climáticas y de cuenca; en nuestro país se ha encontrado una relación entre el $\mathrm{Q}_{347}$ y un caudal obtenido a partir de caudales mensuales de los meses del estiaje (Mora Alonso-Muñoyerro, 1995).

Puede ser interesante a la hora de estimar las fluctuaciones de caudal, el estudio de las características de cuenca y el clima con su influencia en los caudales circulantes; además también se pueden relacionar con los caudales mínimos, en lo que puede derivarse como una metodología para la estimación de los caudales de mantenimiento, que serían los que permiten que el río continúe funcionando permitiendo el mantenimiento de las comunidades biológicas, o los también llamados caudales ecológicos por algunos autores y que normalmente aparecen con esa denominación en los planes de cuenca (Palau, 1994).

Por otro lado estas relaciones pueden servir para explicar el funcionamiento del río en las situaciones de aguas bajas (O’Shea, 1995).
Para la estimación de unos caudales circulantes mínimos que deben mantenerse después de una actuación hidráulica, han venido usándose tres grupos distintos de métodos:

- El análisis de los caudales históricos circulantes.

- Las variaciones que el caudal produce en las variables hidráulicas del cauce inundado y por lo tanto en el hábitat de las comunidades acuáticas.

- La relación entre los caudales circulantes y el hábitat potencialmente utilizable, atendiendo a las necesidades de hábitat de las especies.

El primer grupo de métodos es el que con más frecuencia se viene utilizando por la sencillez de su estimación; una revisión de varios de estos métodos se puede obtener en Mora AlonsoMuñoyerro (1995). Existen intentos de desarrollar nuevos métodos hidrológicos con más sentido biológico y ya se han desarrollado otras nuevas metodologías (Palau, 1994; Palau \& Alcazar, 1996; Baeza \& García del Jalón, 1997).

Con respecto a los otros métodos, White (1976) utiliza el estudio de la forma en la que el caudal varia el perímetro mojado del cauce, asumiendo que el aumento de este produce un aumento también de la capacidad biogénica del río lo que sería un método del segundo tipo. Por último la relación entre los caudales circulantes y las necesidades de hábitat de las especies ha desembocado en la metodología IFIM (Stalnaker, 1979; Bovee, 1982), que está siendo ampliamente utilizada en Norteamérica y también se ha trabajado ya con ella en nuestros ríos (García de Jalón, 1990; Cubillo et al., 1990; Mayo et al., 1993).

En estos métodos no se utiliza ningún estudio de la cuenca, de sus dimensiones y factores meteorológicos, y de los parámetros de esta que puedan suponer oscilaciones en el caudal, aportaciones, salidas y situaciones extremas.

Utilizando la metodología desarrollada por Baeza \& García del Jalón (1997) para la estimación del caudal ecológico en 16 ríos de la cuenca del Tajo, en este trabajo se han estudiado varias características de la cuenca, como son la geomorfología, los tipos de uso y el clima, para 
encontrar algún modelo que sirviera para examinar las relaciones entre estas variables, y el caudal recomendado.

La profundización en estos modelos puede llevarnos a encontrar unas técnicas para el cálculo de estos caudales, en las que se consideren otros factores distintos a los propios caudales históricos que han circulado por del río.

\section{METODOLOGÍA}

Para facilitar la búsqueda de relaciones entre el caudal y las variables de cuenca se ha realizado una sectorización de las cuencas para encontrar tramos fluviales homogéneos, basándose en los diferentes regímenes de caudales naturales.

Esta sectorización fue completada con mapas de vegetación potencial y geológicos (Mapa Geotectonico General, 1976; Mapa de Aprovechamientos y Cultivos, 1977; Mapa de Estados Erosivos de la Cuenca del Tajo, 1977), para una mejor caracterización del entorno del río y fundamentar más la sectorización; de esta forma se han podido sacar modelos que se ajustan a zonas fluviales de características hidrológicas similares.

La presencia de actuaciones hidráulicas en la mayoría de los tramos de la cuenca del Tajo limitó las estaciones de estudio, ya que solamente se trabajó en aquellas con regímenes naturales por encima de aprovechamientos y grandes presas.

Las estaciones de aforo de las que se tomaron los datos están distribuidas por toda la cuenca desde la más occidental la del río Almonte en Monroy hasta la más oriental del Tajo en Peralejos (CEDEX,1994). En total se utilizaron 16 estaciones con datos hidrológicos en una serie histórica suficientemente grande. En este grupo existen ríos de grandes diferencias geomorfológicas, de regímenes, así como tipología de cuenca y zonas climáticas.

\section{Parámetros hidrológicos}

Se evaluaron tres métodos distintos para calcular caudales mínimos. En el primero se utilizó el que denominamos Q25d, este caudal se obtiene ordenando cronológicamente la serie histórica de caudales diarios y calculando la media de los caudales que han circulado durante veinticinco días seguidos; moviendo esta media por toda la serie se obtienen 340 medias de los caudales de veinticinco días consecutivos de todo el año; posteriormente se averigua el mínimo para cada año de esta serie de caudales de 25 días, con eso obtenemos el caudal mínimo de la media de 25 días en cada uno de los años estudiados.

Para obtener un único valor de caudal, de todos los mínimos de todos los años se encuentra la media, y ese será un valor que representa a los caudales que normalmente han circulado por el río durante 25 días de estiaje, en la serie estudiada.

Este es un valor que tiene más sentido biológico que otras medidas utilizadas para estimar caudales ecológicos, ya que este valor de caudal ha circulado por el río durante un periodo largo de días y no es un caudal puntual de un único día, por lo que las comunidades biológicas, lógicamente, deben estar más adaptadas a él.

La metodología de medias móviles se ha utilizado ampliamente en hidrología, para caracterizar series de caudales (Gustard et al., 1992). La elección de un número de días mayor o menor viene determinado por lo que se quiera calcular, en nuestro caso la elección de un intervalo de 25 días es el resultado del análisis de gráficas (caudal $/ \mathrm{n}^{\circ}$ de días); en estas gráficas (Fig. 1) hemos comprobado que, aumentando el número de días en los que calculamos el caudal, por medio de medias móviles, no se producen aumentos significativos de cual a partir de 25 días, produciéndose una estabilización de valores alrededor de este valor.

Este resultado lo hemos interpretado como una estabilización de los caudales en muchos ríos, que establece la cuenca en el estiaje, siendo muy infrecuentes los valores de caudal menores a los que se obtienen en esos días, para un mejor desarrollo de este método se puede consultar Baeza \& García del Jalón (1997).

Los otros dos métodos se basan también en la ordenación cronológica de los caudales diarios y el cálculo de medias móviles pero en este caso se calculan para un número de días que va crecien- 


\section{Río Guadiela}

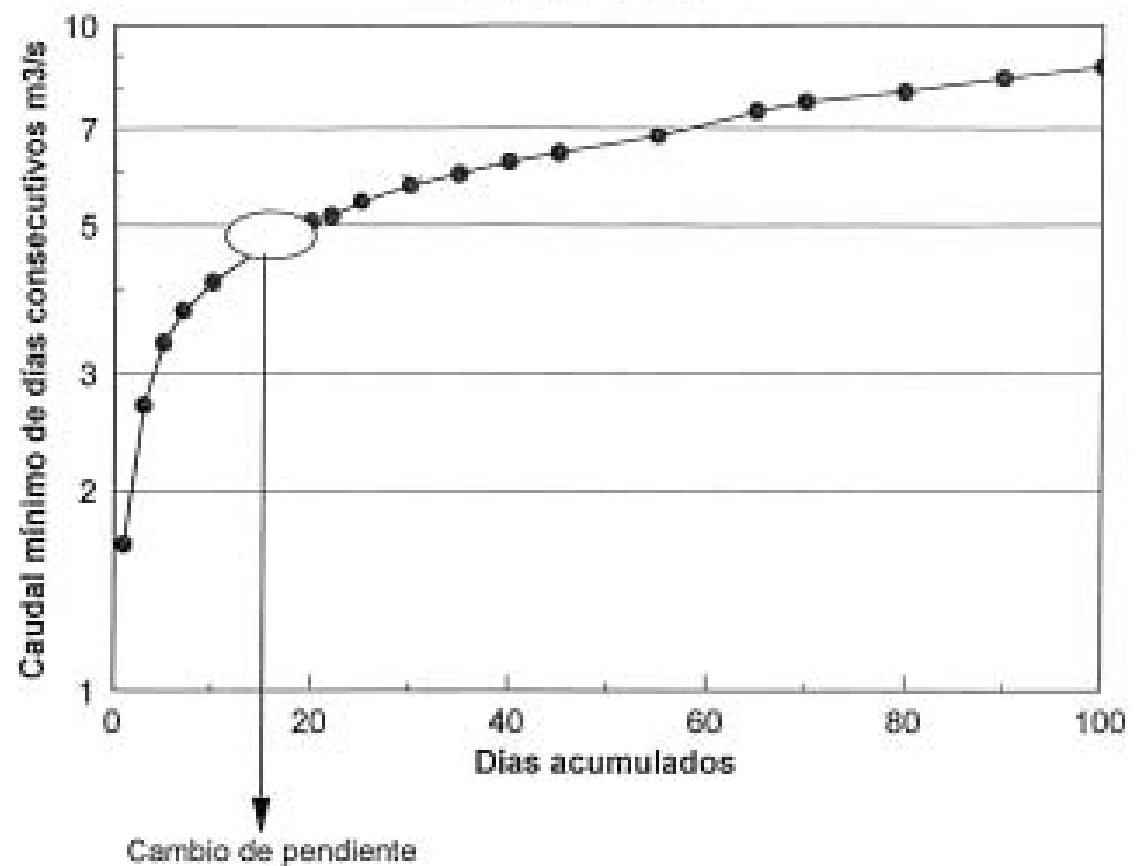

Rio Jerte

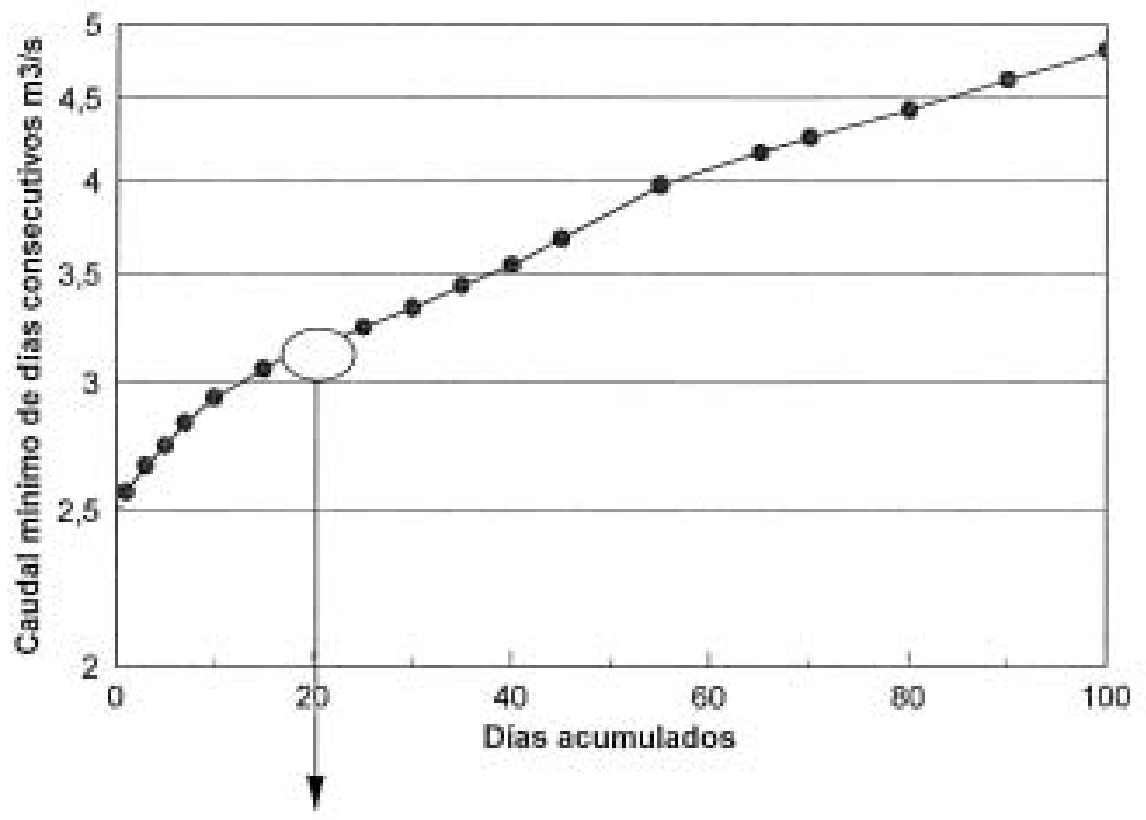

Cambio de pendiente

Figura 1. Representación de la variación de caudal con respecto al número de días utilizado para su cálculo; el cambio de pendiente se observa mejor en el río Guadiela que en el Jerte. 
do obteniéndose series de medias para uno, cuatro, siete,...etc., número de días en incrementos de tres días hasta llegar a cien. Posteriormente se calcula la pendiente de cada tramo y se averigua la pendiente máxima, tomando como caudal aquel de los dos el mayor en los que se encontró la pendiente máxima de su tramo, este método se describe en CEDEX (1998). A este caudal lo hemos llamado Qbase.

En el ultimo método utilizando los datos de caudales para distinto número de días, se traza la gráfica en la que se representa el caudal, frente a los días utilizados para calcular las medias móviles. Estas gráficas presentan varias tipologías descritas en Baeza \& García del Jalón (1997), cóncavas, convexas, de monotonía creciente y con varios cambios de pendientes. Se toma como caudal mínimo aquel que corresponde a un punto característico de la curva, bien donde se produce un cambio de pendiente o dónde pasa la curva de cóncava a convexa; este punto se aprecia subjetivamente. A este valor lo hemos denominado Qman.

\section{Parámetros de la cuenca}

En cuanto a las variables de cuenca que utilizamos para contrastar con estos, se han incluido dos parámetros hidrológicos, que son el caudal medio y el coeficiente de escorrentía.

Características propias de la cuenca han sido once; de estos, cinco corresponden a factores que influyen en la erosión de la cuenca que son: el coeficiente R (índice de erosión pluvial), $\mathrm{K}$ (factor de erosionabilidad del suelo), C factor cultivo, LS factor pendientes de la cuenca y el A (perdida de suelo). Otros fueron: la superficie de la cuenca, el porcentaje de cuenca cubierto por superficie forestal (Segundo Inventario Forestal Nacional, 198695) y el porcentaje de tierras agrícolas.

Por último otros tres climáticos que son, la temperatura media, la precipitación, la evapotranspiración potencial (ETP).

También a partir de los datos de superficie forestal y agrícola y las características de los suelos y su permeabilidad se calcularon los números

Table 1. Nomenclatura utilizada en los modelos para nombrar a las variables de cuenca estudiadas, y las unidades en las que se miden. Nomenclature used in models for naming the basin variables studied, and units in which they were measured.

\begin{tabular}{lcc}
\hline Nombre & Símbolo & Unidades \\
\hline Indice de erosión pluvial & $\mathrm{R}$ & $\mathrm{J} / \mathrm{m}^{2} \mathrm{~cm} / \mathrm{hora}$ \\
Factor de erosinabilidad del suelo & $\mathrm{K}$ & $\mathrm{m} 2 \mathrm{hora} / \mathrm{ha} . \mathrm{J} . \mathrm{cm}$ \\
Perdida de suelo & $\mathrm{A}$ & Ton/ha \\
Factor cultivo & $\mathrm{C}$ & $\%$ \\
Factor topográfico & $\mathrm{LS}$ & $\%$ \\
Precipitación & Pre & $\mathrm{mm}$ \\
Temperatura & Temp & ${ }^{\circ} \mathrm{C}$ \\
Evapotranspiración & ETP & $\mathrm{mm}$ \\
Superficie & Superficie & $\mathrm{km}$ \\
Caudal medio & Qmedia & $\mathrm{m}^{3} / \mathrm{s}$ \\
Escorrentia & Escorre & $\%$ \\
Forestal arbolado & F.A. & $\%$ \\
Forestal ralo & F.R. & $\%$ \\
Forestal desarbolado & F.D. & $\%$ \\
Cultivo & Cultivo & $\%$ \\
$\%$ Cabida cubierta & Cab. c. & $\%$ \\
N de Curva & N deC & $\mathrm{mm}^{-1}$ \\
Pendientes & Pend & $\%$ \\
q25d & q25d & $\mathrm{m}^{3} / \mathrm{s}$ \\
q25dm & q25dm & - \\
\hline
\end{tabular}


hidrológicos de curva, tal y como se describen en el método de United States Department of Agriculture (U.S.D.A.) (1978).

Los datos de aforos se han tomado de los libros de aforos publicadas por la Confederación Hidrográfica del Tajo, los demás datos se obtuvieron de todas las estaciones meteorológicas mas incluidas en las cuencas y las más cercanas a ellas.

Para los datos de valores de erosión se tomaron los establecidos en la red de puntos, en los que se midieron caracteres, para elaborar el mapa de estados erosivos de la cuenca del Tajo, de aquellos situados más próximos a las cuencas.

Con respecto a los valores para obtener el número de curva, se tomaron datos del citado inven- tario forestal, del Mapa Geotectónico General del Instituto Geológico y Minero (1977) y del Mapa de Aprovechamientos y Cultivos (1977). Cuando la cuenca por encima de la estación de aforo era muy extensa, se tomaron datos de dos o más estaciones, y se calculo la media. Los parámetros y sus unidades se encuentran en la Tabla 1.

Como ya se ha mencionado, también se revisaron los mapas de vegetación potencial y los geológicos para una mejor caracterización del entorno del río y fundamentar más la sectorización, aunque, en este caso no se pudieron expresar las variables tomadas de forma cuantitativa.

Primero se busco la correlación entre los tres caudales de mantenimiento obtenidos y se esta-

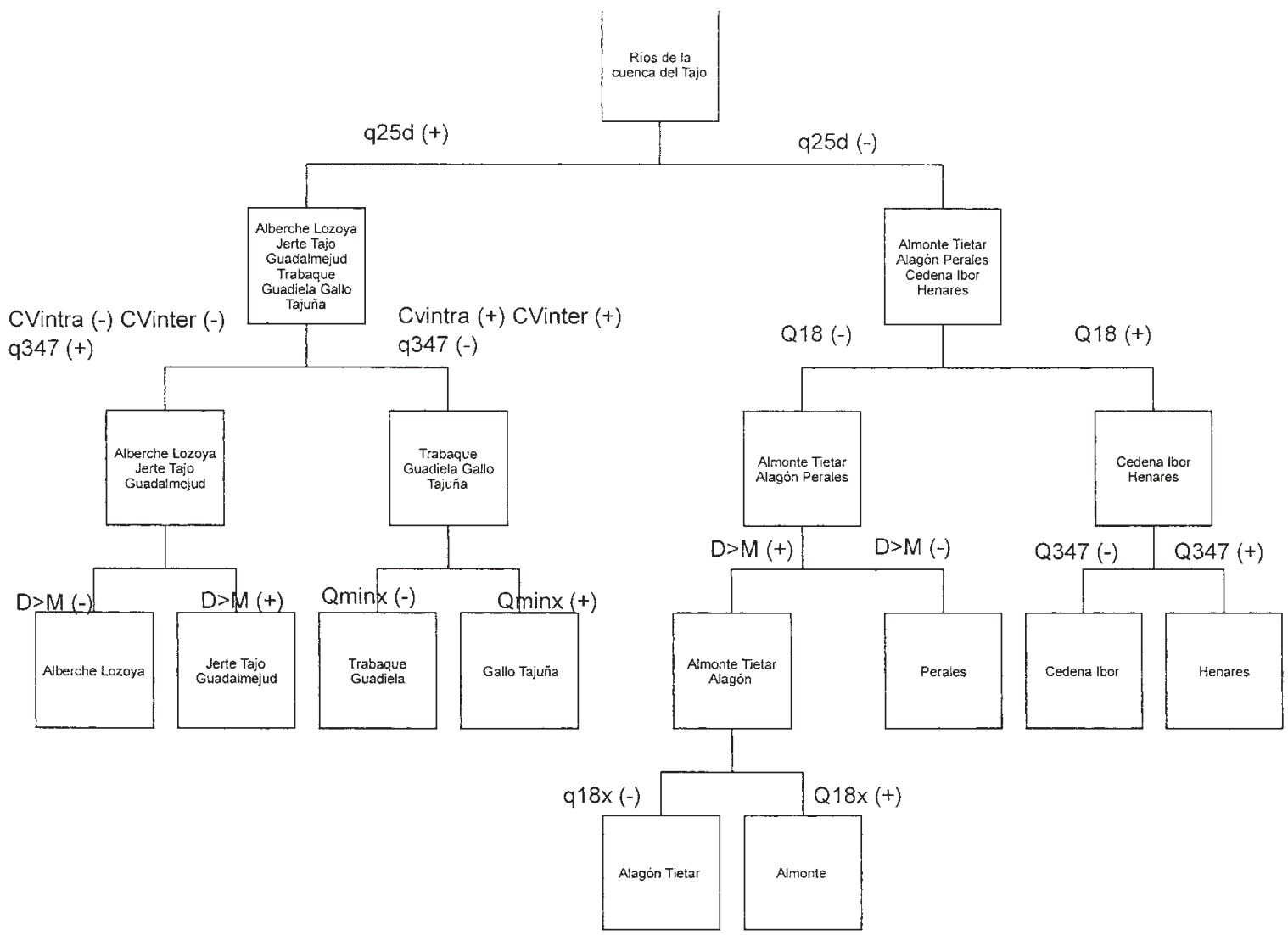

Figura 2. Clasificación de los 16 ríos utilizados en el estudio obtenida con el programa TWINSPAN, indicando las variables de mayor peso para separar los grupos. Classification of the 16 rivers studied using TWINSPAN program. Main variables are indicated.Change in flow estimates in relation to the number of days used for calculation. Slope change is clearer in River Guadiela than in the River Jerte. 
bleció trabajar solo con uno de ellos si se presentaba una alta colinerialidad.

Una vez establecido el caudal con el que se quería trabajar y con los valores de las variables de cuenca obtenidos, se realizó un análisis de regresión múltiple por pasos para examinar la relación entre Q25d y estas; posteriormente se calculó el coeficiente de determinación $\mathrm{r}^{2}$, para comprobar que parte de la varianza del parámetro se explica con los modelos obtenidos.

Para comprobar si existía alguna correlación basada en el orden en el que se encontraban los datos, se aplicó un test de Durbin-Watson a los residuos, y se realizó una representación gráfica de estos para observar algún problema que violara las regresiones asumidas.

El caudal obtenido Q25d, se dividió entre el módulo obteniendo un nuevo parámetro que denominamos Q25dm; este sirvió para comprobar el porcentaje del módulo que representa este caudal y determinar si este caudal mínimo está muy alejado de los valores medios del agua que lleva el río.

El estudio estadístico se aplicó primero al conjunto de los 16 ríos y luego, separadamente, a tres subgrupos que se hicieron con ellos, después de realizada una sectorización en base a características de sus regímenes.

Estos grupos se han tomado así basándose en una clasificación realizada con el programa Twinspan (Hill, 1979), en la que se tomaron en cuenta sólo valores referentes a sus regímenes (Fig. 2); los resultados de este trabajo están en Baeza \& García del Jalon (1997).

En un primer grupo se encuentran los ríos Tajo, Guadamejud, Guadiela, Trabaque, Tajuña y Gallo, que son los ríos de los estudiados con características de regímenes más iguales, y una proximidad geográfica que hace que coincidan muchas características de sus cuencas. Estos ríos tienen un caudal predecible y sus caudales de estiaje son altos comparándolos con los de los otros días a demás de que el estiaje es de corta duración.

El segundo grupo de ríos estuvo formado por los seis del grupo anterior a los que se añadió el Alberche, el Lozoya y el Jerte. En este grupo se

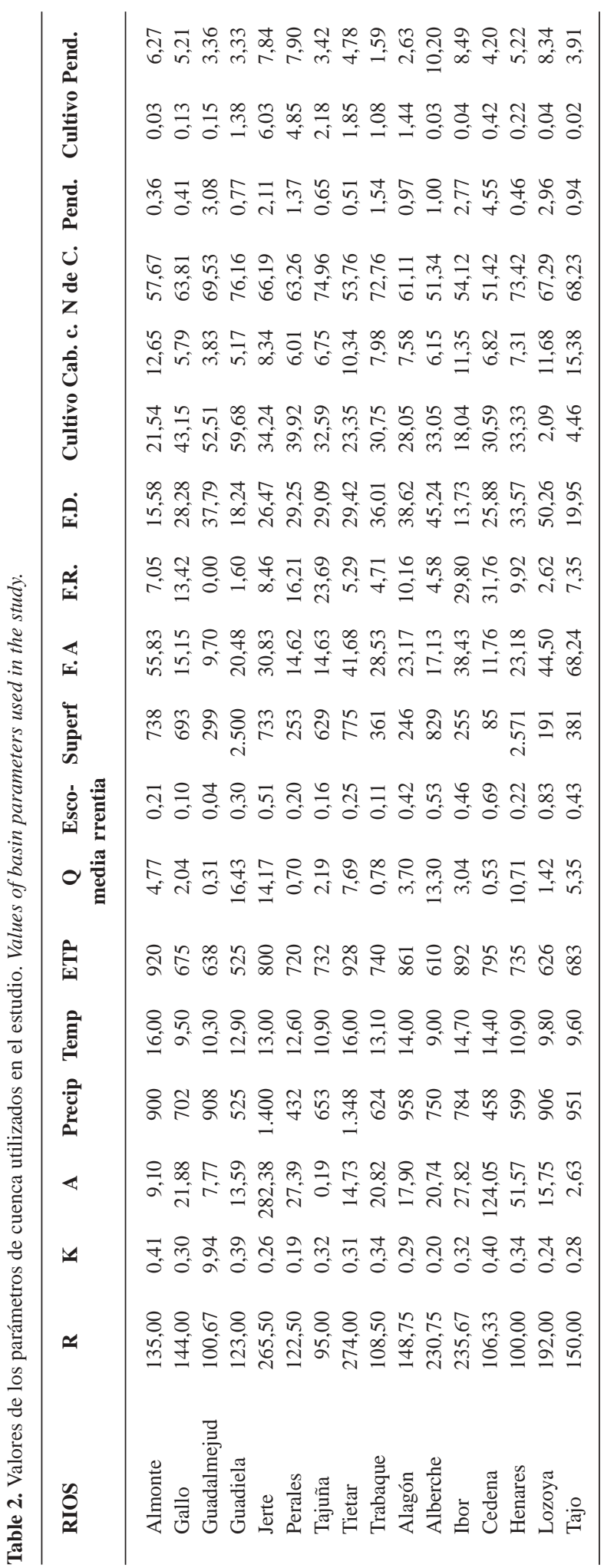


quiso ver el comportamiento de aquellos ríos que tienen algún tipo de regulación natural, bien sea de tipo nival o geológico, aparte de que las características de sus regímenes son más próximas que las de los restantes.

El tercer grupo comprende el Almonte, Tietar, Alagón, Perales, Cedena, Ibor y Henares. Estos tiene caudales de estiaje bajos en comparación al grupo; en general los caudales son poco predecibles, aunque este es un grupo menos homogéneo que los anteriores.

En cada uno de estos grupos se hizo un análisis de correlación múltiple, como se hizo con el grupo que comprendía a todos ellos, utilizando Q25d con los dos primeros grupos y Q25dm con el último.
Para este análisis se ha utilizado el paquete estadístico STATGRAPHICS en su versión 2.1.

\section{RESULTADOS}

En la Tabla 2 se encuentran los resultados de los valores obtenidos para las variables de cuenca en los 16 ríos, y que se han utilizado posteriormente para el estudio estadístico.

Las características de vegetación potencial y de geología, no se han incluido en el análisis estadístico pero han servido para complementar la sectorización obtenida con anterioridad, una sencilla clasificación de las formaciones vegetales predominantes en las cuencas se encuentra en la Tabla 3.

Table 3. Relación entre pisos y series de vegetación con los ríos que comparten parte de la cuenca. Relationship between vegetation levels and sub-levels, with rivers that have total or partially the same basin.

\begin{tabular}{|c|c|c|}
\hline Piso Supramediterraneo & & \\
\hline Ambito geológico & Silicícola & Calcícola \\
\hline Ríos & Lozoya & Tajo \\
\hline \multicolumn{3}{|l|}{ Piso Oromediterraneo } \\
\hline Ambito geológico & Silicícola & Calcícola \\
\hline \multicolumn{3}{|l|}{ Régimen de humedad y especies } \\
\hline Humedo (Quejigares y melojares) & $\begin{array}{l}\text { Lozoya, Jerte, Alberche, Alagón, } \\
\text { Cedena, Ibor, Tietar }\end{array}$ & $\begin{array}{l}\text { Henares, Tajo, Gallo, Guadiela, } \\
\text { Trabaque, Tajuña }\end{array}$ \\
\hline Seco (Encinares y Sabinares) & Perales, Tietar & $\begin{array}{l}\text { Gallo, Tajo, Tajuña (Sabina)Trabaque, } \\
\text { Guadiela, Henares (Encina) }\end{array}$ \\
\hline \multicolumn{3}{|l|}{ Piso Mesomediterraneo } \\
\hline Ambito geológico & Silicícola & Calcícola \\
\hline \multicolumn{3}{|l|}{ Régimen de humedad y especies } \\
\hline Húmedo (Melojares y Alcornocares) & $\begin{array}{l}\text { Jerte (Roble melojo) Alagón, } \\
\text { Ibor (Alcornoque) }\end{array}$ & \\
\hline Seco (Encinares) & Almonte Ibor, Cedena, Tietar & Guadamajud, Trabaque \\
\hline
\end{tabular}


Table 4. Caudales mínimos ecológicos en $\mathrm{m}^{3} / \mathrm{s}$ calculados con los tres estimadores y sus coeficientes de correlación. Minimum, ecological, flows $\left(\mathrm{m}^{3} / \mathrm{s}\right)$ calculated from the three estimators and their correlation coefficients.

\begin{tabular}{lrrr}
\hline RIOS & Q25d & Qman & Qbase \\
\hline Almonte & 0,024 & 0,960 & 0,87 \\
Gallo & 0,886 & 1,096 & 1,12 \\
Guadamejud & 0,071 & 0,088 & 0,11 \\
Guadiela & 5,419 & 13,410 & 13,69 \\
Jerte & 3,243 & 5,820 & 4,88 \\
Perales & 0,011 & 0,039 & 0,04 \\
Tajuña & 0,800 & 0,970 & 0,95 \\
Tietar & 0,064 & 0,350 & 0,14 \\
Trabaque & 0,206 & 0,282 & 0,30 \\
Alagón & 0,190 & 0,490 & 0,29 \\
Alberche & 2,250 & 6,390 & 6,47 \\
Ibor & 0,350 & 0,440 & 0,44 \\
Cedena & 0,100 & 0,120 & 0,13 \\
Henares & 0,370 & 1,510 & 0,99 \\
Lozoya & 0,150 & 0,320 & 0,23 \\
Tajo & 1,310 & 1,730 & 1,77 \\
\multicolumn{2}{c}{ Correlación } & Qbase & Qman \\
\hline & Q25d & $\mathbf{0 , 9 6 5 3}$ & $\mathbf{0 , 9 7 4 4}$ \\
\hline & Qbase & $\mathbf{0 , 9 9 7 0}$ \\
\hline
\end{tabular}

\section{Variables no cuantitativas}

Las series de vegetación potencial se han estudiado puesto que la vegetación potencial es un indicador de características edáficas y climáticas de las áreas geográficas donde se encuentran, y pueden ayudar a explicar la inclusión de los ríos en los grupos y la sectorización basada en razones de características que se encuentran en las porciones de cuenca existentes por encima de las estaciones de aforo de cada río.

Todos las superficies están en pisos mesomediterraneos, supramediterraneos y oromediterraneos.

Empezando por los de mayor altitud, sólo tienen parte de su cuenca en pisos oromediterraneos el Lozoya en una zona silicícola y el Tajo en una calcícola.

En el piso supramediterraneo, en la serie de los melojares mediterraneos, hay cuencas de los ríos Lozoya, Tajuña, Jerte, Alagón Tietar, Alberche e
Ibor. En la serie de los quejigares el Henares, Tajo, Gallo, Guadiela, Trabaque y Tajuña. En los sabinares albares el Tajo y el Tajuña. En las series de los encinares corresponde a la serie basófila de la encina parte de la cuenca del Trabaque y del Henares y en la silicícola de la encina hay una superficie de cuenca de los ríos Tietar y Perales.

En cuanto al piso mesomediterraneo, en la serie de los melojares y quejigares tienen parte de sus cuencas los ríos Jerte y Alagón, el Ibor en la serie de los alcornocales y en las series de encinares el Guadamajud, Trabaque en la parte basófila y el Almonte e Ibor en la silicícola.

En cuanto a las características geológicas son similares para el Gallo, Tajuña, Guadamejud, Tajo, Guadiela y Trabaque; en los que predominan las

Table 5. Coeficientes de correlación encontrados para las variables de mayor significación. Correlation coefficients found for the main variables of greatest significance.

\begin{tabular}{lcc}
\hline Conjunto completo de ríos: & \\
& Qmedia & Superficie \\
\hline Q25d & 0,8226 & 0,5383 \\
\hline
\end{tabular}

$1^{\text {er }}$ grupo de ríos: Tajo, Tajuña, Gallo Guadiela, Guadamejud y Trabaque.

\begin{tabular}{ccccc} 
& Qmedia & Superficie & F.R. & ETP \\
\hline Q25d & 0,9886 & 0,9464 & $-0,8547$ & $-0,7974$ \\
\hline
\end{tabular}

$2^{\circ}$ grupo de ríos: Tajo, Tajuña, Gallo Guadiela, Guadamejud, Trabaque, Alberche, Lozoya y Jerte

\begin{tabular}{ccc} 
& Qmedia & Superficie \\
\hline Q25d & 0,9348 & 0,8731 \\
\hline
\end{tabular}

$3^{\circ}$ grupo de ríos: Almonte, Tietar, Alagón, Perales, Cedena, Ibor y Henares

\begin{tabular}{cccc} 
& Escorren & A & Pend \\
\hline Q25dm & 0,9608 & 0,8217 & 0,9668 \\
\hline
\end{tabular}


calizas, dolomitas, areniscas, margas y conglomerados. El Henares tiene una cuenca de composición similar pero aparecen yesos y margas yesíferas.

Las rocas ígneas y metamórficas son dominantes en las cuencas del Tietar, el Almonte con pizarras, Jerte y Alberche, así como el Alagón y el Lozoya.

En el río Perales los materiales están más sueltos; existen arcosas, gravas y cantos de gneiss y granitos muy sueltos. Cedena e Ibor se asientan sobre areniscas cuartillas y pizarras. Estas distribución concuerda bastante con los grupos que se han establecido.

\section{Análisis estadístico}

El análisis estadístico comenzó encontrando los coeficientes de correlación de los tres estimadores usados, el Q25d, Qbase, y Qman, entre si, los valores obtenidos para estos tres se encuentran en la Tabla 4.

Se comprobó que entre los tres estimadores existía una alta colinerialidad, por lo que de aquí en adelante sólo se trabajó con Q25d (Tabla 4).

Posteriormente se calcularon los coeficientes de correlación entre el caudal Q25d y los parámetros de cuenca y climáticos.

Una vez obtenidos los valores de la correlación y su significación, se tomaron aquellos valores de los estudiados que tuvieran una significación estadística al 95\% de nivel de confianza, considerando que son los que más importancia tienen en la variabilidad de estos parámetros

\section{Análisis de correlación}

Cuando se realiza una tabla de correlación entre los valores del caudal Q25d y los demás datos del estudio, utilizando todos los ríos (Tabla 5), se observa que, de las variables de cuenca, están más fuertemente correlacionados con Q25d, el caudal modular (Qmedia) y la superficie de la cuenca (Superficie), con valores $\mathrm{r}=0,8226(\mathrm{p}=$ $0,0001)$ y $r=0,5383(\mathrm{P}=0,0055)$.
Analizando el primer grupo de ríos, los seis de la cabecera del Tajo, las correlaciones más fuertes aparecen con F.R. (forestal ralo) $(\mathrm{r}=-0,8547, \mathrm{P}$ $=0,0301, \operatorname{ETP}(\mathrm{r}=-0,7974, \mathrm{P}=0,0341)$, Qmedia $(\mathrm{r}=0,9886, \mathrm{P}=0,0001)$ y Superficie $\mathrm{r}$ $=0,9464(\mathrm{P}=0,0009)$.

En el segundo grupo de ríos, en el que se incluían el Alberche, Lozoya y Jerte a los seis del primero, se han encontrado las mayores correlaciones con Qmedia ( $\mathrm{r}=0,9348, \mathrm{P}=0,0002)$ y Superficie $(r=0,8731, P=0,0021)$, ahora con valores del coeficiente de Pearson más bajos.

En el tercer grupo de ríos, las correlaciones mayores fueron con Qmedia, Superficie y el número de días empleados para calcular el Qbase, pero con valores bastante bajos y con poca significación estadística. Por el contrario, en este último grupo se ha obtenido una correlación alta entre el Q25d/m, que corresponde al caudal q25d dividido entre el caudal modular, con la escorrentía (Escorren) $(r=0,9608$, $\mathrm{P}=0,0007)$, las pendientes del río (Pend) $(\mathrm{r}=0,9668, \mathrm{P}=0,0004)$ y la pérdida de suelo (A) $(r=0,8217, P=0,0207)$.

\section{Los modelos}

A) Global: En el primer modelo utilizando todos los ríos, el mejor modelo lineal encontrado relaciona Q25d y las variables Qmedia y Superficie según la siguiente ecuación:

Q25d $=-0,0427+0,222 \times$ Qmedia - 0,0004 x Superficie

Este modelo tiene un error standard de 0,6643 $\mathrm{m}^{3} / \mathrm{s}$, su coeficiente de determinación $\mathrm{r}^{2}$ es del $71 \%$; y su valor $\mathrm{P}=0,0007$, tiene una significación estadística al $99 \%$. El estadístico de Durwin-Watson índica que no existe autocorrelación entre los residuos.

En él análisis por pasos se puede ver que el valor de $\mathrm{P}$ es muy alto $(\mathrm{P}=0,5827)$ para Superficie y no tiene significación estadística al $90 \%$, por lo que el modelo se puede simplificar, eliminando esta variable. 
Cuando se representan los valores observados frente a los obtenidos por el modelo, se comprobó que algunos de estos últimos son negativos, y que corresponden a aquellos ríos con Qmedios muy bajos, lo que cuestiona la validez del estimador Q25d para estos ríos.

Con objeto de evitar los valores negativos recurrimos a un modelo polinómico de grado dos, en el que el coeficiente de determinación es $\mathrm{r}^{2}$ $=89,05$ y error $0,533 \mathrm{~m}^{3} / \mathrm{s}$; el valor de la significación para el grado dos es suficiente para un nivel de confianza del $99 \%$, y por lo tanto este grado es suficiente para explicar la relación existente entre los dos parámetros. El modelo encontrado es el siguiente:

$\mathrm{Q} 25 \mathrm{~d}=-0,4017+0,972 \times$ Qmedia - 0,278 $\mathrm{x}$ Qmedia $^{2}$

B) Primer grupo: En el primer grupo de ríos, con el estudio de correlación múltiple por pasos, se obtuvo que el mejor modelo era aquél en el que se elimina la variable ETP (evapotranspiración), y por lo tanto sólo utiliza F.R., Qmedia y Superficie, el modelo encontrado es:

Q25d $=1,704-0,049 \times$ F.R. + 0,062 x Qmedia $+0,0007$ Superficie

Este modelo tiene un $\mathrm{r}^{2}=99,98$, un error estándar de $0,03 \mathrm{~m}^{3} / \mathrm{s}$ y el valor $\mathrm{P}=0,0004$ es significativo al $99 \%$

Cuando trabajamos con el segundo grupo de ríos, en el modelo que mejor se ajusta a las observaciones es el que se construye con Qmedia y con Superficie.

Q25d $=-0,113+0,182 \times$ Qmedia $+0,0002 \times$ Superficie

Este modelo tiene un coeficiente de determinación $\mathrm{r}^{2}=95,54$, tiene significación estadística al $99 \%$ y un error de $0,32 \mathrm{~m}^{3} / \mathrm{s}$.

C) Tercer grupo: Por último, en el tercer grupo de ríos, las correlaciones obtenidas de los valores del caudal Q25d con las variables son muy bajas y de poca significación estadística; no es así cuando se trabajó con Q25dm, por tanto el modelo se construyó con esta variable como variable independiente. Se encontró una alta correlación con las variables A (factor pérdida de suelo), Pend (pendientes del río) y Escorren (coeficiente de escorrentía), pero el mejor modelo es el que sólo utiliza las dos últimas:

$\mathrm{Q} 25 \mathrm{dm}=-0,0396+0,176 \times$ Escorren $+0,024 \mathrm{x}$ Pend

El coeficiente de determinación es $\mathrm{r}^{2}=91,46$, el error de la estimación de $0,021 \mathrm{~m}^{3} / \mathrm{s}$ y tiene significación al $99 \%$.

Los resultados y estadísticos de los modelos se encuentran en la Tabla 6, así como las gráficas en la figura 3.

\section{DISCUSIÓN Y CONCLUSIONES}

El método elegido para estimar los caudales mínimos, que consiste en medir caudales bajos que han estado circulando por el río durante 25 días en el estiaje, tiene más base biológica que aquellos en los que se utilizan caudales puntuales o porcentajes fijos del módulo (Tennant, 1976); en este método se ha supuesto una adaptación de las poblaciones a un periodo largo de tiempo de caudales bajos continuados.

Por el contrario, si el caudal que se deja como ecológico es un caudal que sólo se presenta excepcionalmente una vez en el río y se mantienen como un continuo durante largos periodos de tiempo, las poblaciones habitantes del río sufrirán una perturbación a la que no están adaptadas y que no serán capaces de superar.

Una posible forma de evaluar la validez de los valores encontrados a partir del cálculo del Q25d es compararlos con el valor del módulo del río. En cinco ríos, Almonte, Perales, Tiétar, Alagón y Henares, el caudal obtenido representa menos del $10 \%$ del valor modular.

Puede ser este un límite muy bajo para mantener determinadas poblaciones en el río, especialmente si se mantiene durante un periodo muy largo; no consideramos que sea válido si el crite- 


\section{MODELO POLINÓMICO INCLUYENDO TODOS LOS RÍOS}

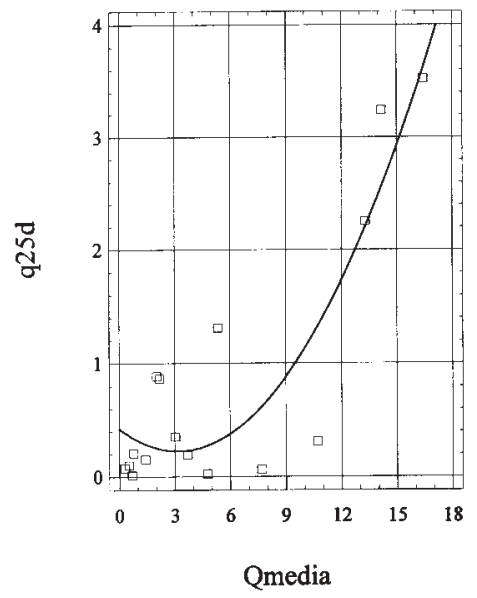

MODELO PARA EL $2^{\circ}$ GRUPO DE RÍOS

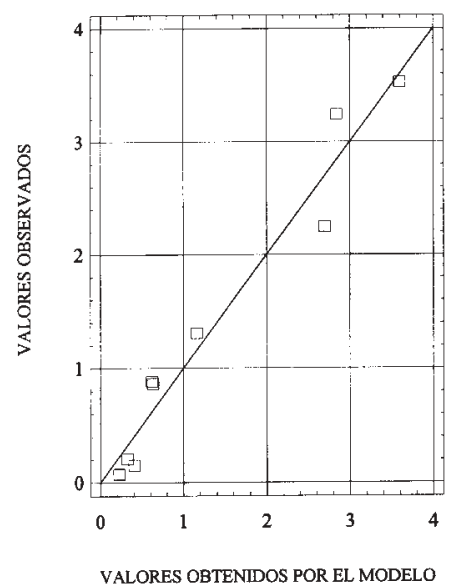

MODELO PARA EL 1ํ GRUPO DE RÍOS

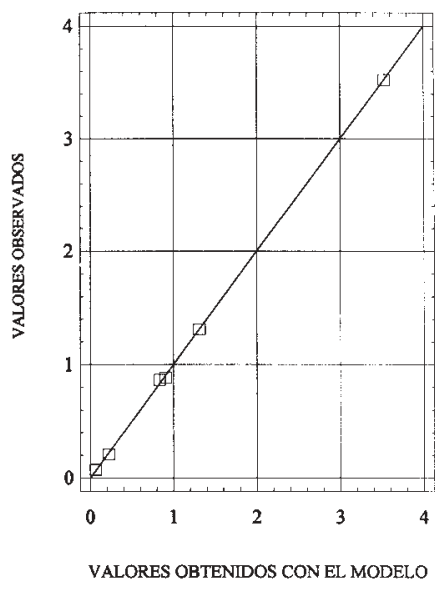

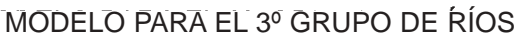

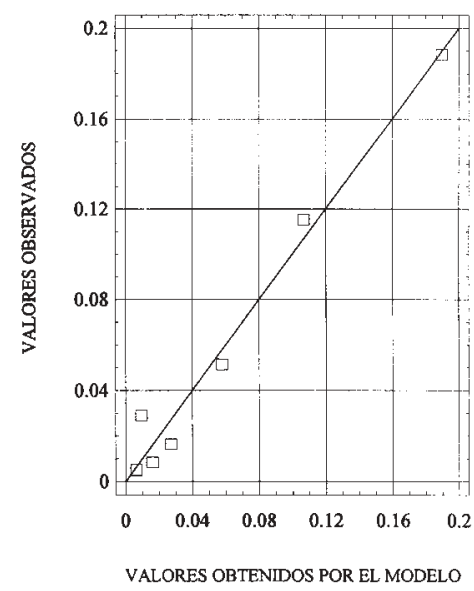

Figura 3. Modelos obtenidos para los grupos de ríos estudiados. Models obtained fot studied groups of rivers.

rio se utiliza como general en todos los ríos, como por ejemplo se usa en la Comunidad de Castilla y León, aunque se puede aplicar, después de una adecuada regionalización, a aquellos ríos con características peculiares de descenso natural de caudales en el estiaje.

Por este motivo en los ríos en los que con 25 días se obtengan caudales bajos, se deberá estudiar cui- dadosamente su comportamiento en el estiaje, y analizar las peculiaridades del régimen, puesto que posiblemente estos valores están en el río solamente unos pocos días, y el régimen sea muy intermitente, presentando caudales muy bajos, pero durante periodos muy cortos.

Esto hace necesario buscar más parámetros que caractericen el régimen y que también presenten 
Table 6. Modelos encontrados y estadísticos utilizados. Models found and statistics used.

\begin{tabular}{|c|c|c|c|c|c|c|}
\hline Grupo de ríos & Modelo & $\mathbf{r}^{2}$ & $\begin{array}{c}\text { Error estándar } \\
\left(\mathrm{m}^{3} / \mathrm{s}\right)\end{array}$ & F-test & Valor de $P$ & $\begin{array}{l}\text { Durwin- } \\
\text { Watson }\end{array}$ \\
\hline $\begin{array}{l}\text { Conjunto } \\
\text { completo }\end{array}$ & $\begin{array}{l}\text { Q25d }=-0,402+0,972 \text { Qmedio } \\
-0,278 \text { Qmedia }^{2}\end{array}$ & 89,05 & 0,533 & 22,36 & $\mathrm{P}<0,01$ & 1,8 \\
\hline Grupo 1 & $\begin{array}{l}\text { Q25d }=1,704-0,049 \text { F.R. } \\
+0,062 \text { Qmedio }+0,0007 \text { Superf }\end{array}$ & 99,97 & 0,029 & 2999 & $\mathrm{P}<0,01$ & 2,49 \\
\hline Grupo 2 & $\begin{array}{l}\mathrm{Q} 25 \mathrm{~d}=-0,113+0,182 \text { Qmedio } \\
+0,0002 \text { Superficie }\end{array}$ & 95,54 & 0,322 & 64,39 & $\mathrm{P}<0,01$ & 1,41 \\
\hline Grupo 3 & $\begin{array}{l}\mathrm{Q} 25 \mathrm{dm}=-0,040 \\
+0,176 \text { Escorren }+0,024 \text { Pend }\end{array}$ & 97,71 & 0,013 & 81,60 & $\mathrm{P}<0,01$ & 1,28 \\
\hline
\end{tabular}

importancia biológica, como los que estiman la frecuencia de cambios de caudales en el río (Richter et. al., 1997); la presencia de este comportamiento fluctuante en estos ríos puede estar relacionado con la forma de abastecimiento de agua al río y el tipo de regulación de su cuenca.

\section{El grupo tres}

Los cinco ríos mencionados antes se agruparon junto al Cedena y el Ibor para formar un subgrupo (Grupo 3) de ríos con características de sus regímenes similares; trabajando con este grupo se ha visto que el Q25dm tiene una alta relación con la pérdida de suelo, es decir con la erosión (factor A) y con la escorrentía; esto puede indicarnos que la cuenca no actúa como regulador de los caudales de estos ríos, y el aporte principal a los cauces proviene del agua de lluvia.

En estos ríos aquellos que tienen valores de Q25dm altos también tienen grandes escorrentías y pérdidas de suelo. El agua de lluvia en verano inmediatamente llega al río y prácticamente nada se infiltra. En los ríos de este grupo con valores de Q25dm bajos lo que ocurre es que la escorrentía es pequeña y la perdida de suelo también lo es; los caudales bajos del río son exiguos, ya que la poca lluvia que cae en el estiaje se infiltra y no contribuye prácticamente nada al caudal del río al ser tomada por un suelo poroso pero vacío.
Todos estos ríos se encuentran en zonas de geología ígnea o metamórfica y tienen parte de sus cuencas en pisos mesomediterraneos de la encina, es decir su vegetación potencial está adaptada a periodos de sequía prolongados; sin embargo, parte de las cuencas del Alagón y Tietar se encuentran en zonas más húmedas, quizás por esta razón en otras clasificaciones también han aparecido juntos y presentan características hidrológicas similares.

El río Cedena e Ibor constituyen una unidad más homogénea dentro de este grupo. Se encuentran sobre materiales detríticos de areniscas, cuarcitas y pizarras. Sus cuencas están en pisos y series de vegetación iguales y, también, son similares los parámetros hidrológicos respecto a la erosión y erosionabilidad del suelo, que son altas.

Un río que aún estando sobre terrenos calizos se encuentra en este grupo es el Henares. El régimen es irregular y su valor Q25d muy bajo; aparentemente el terreno no actúa regulando los caudales bajos, como ocurre con los otros ríos calizos estudiados. De todas formas el terreno es menos uniforme pues presenta yesos y margas yesíferas en su cuenca.

Es interesante destacar que el Henares es uno de los ríos con mayor superficie de cuenca hasta la estación de aforos, y por lo tanto en la que más variables del medio pueden entrar en juego así como un mayor número de afluentes, que pueden modificar el régimen del río. 


\section{Grupo general, con los 16 ríos}

Cuando se trata el conjunto de los dieciséis ríos, aparece como variable más significativa el caudal modular; pensando en la forma de estimar el Q25d no resulta intuitiva esta relación, ya que un río con un caudal modular alto puede tener periodos de aguas bajas largos, y viceversa.

Si tiene, sin embargo, una explicación hidrológica; el caudal medio del río representa el resultado de una serie de acontecimientos de toda la cuenca, que mantienen esa cantidad de agua en el río, haciendo este más o menos caudaloso; estos fenómenos de mantenimiento del agua en el río, pueden ser los mismos que no permitan al río bajar de caudal de forma tremenda, durante un periodo largo de días, que es lo que expresa el Q25d.

El modelo encontrado, polinómico de segundo orden, sirve para todos los ríos, incluso los de caudales muy bajos y permite hacer una primera estimación de un caudal mínimo, de cualquier río, mediante un dato muy fácil de obtener, su caudal medio.

\section{El grupo dos}

La otra variable que toma importancia es la superficie de la cuenca; esta variable es significativa sólo en el grupo dos en el que se encuentran Alberche, Lozoya, Jerte, Tajo, Guadamejud, Trabaque, Guadiela, Gallo y Tajuña.

Si la cuenca es mayor, los mecanismos de regulación se potencian, actúan en una mayor superficie y el control es por lo tanto mayor.

Son varias las razones que indican que este grupo de ríos tiene una alta regulación de sus caudales, por las características de la cuenca, y por lo tanto la superficie de esta representa una variable fundamental para explicar los cambios que se producen en estos.

Encontramos que tanto los tres primeros, debido a la nieve de sus montañas, como los seis últimos, debido al aporte de sus acuíferos calizos, tienen una fuente de mantenimiento de sus caudales, que hace que los caudales muy bajos sean acontecimientos muy improbables y que no se mantengan durante un periodo largo, sino que sean de corta duración y muy puntuales.

Los tres primeros ríos de este grupo, Jerte, Alberche y Lozoya, tienen la cota máxima de su cuenca por encima de los 2000 m; además también presentan en común un alto índice de erosión pluvial y la escorrentía que también lo es (superior al $50 \%$ ); estas variables pueden presentar estos valores debido a su situación geográfica, y a la forma de precipitación en las regiones montañosas.

\section{El grupo 1}

Los ríos que comprenden este grupo son los seis ríos calizos que son los más parecidos en sus regímenes y en sus cuencas; en este grupo hay cuatro variables que están altamente relacionadas con su caudal Q25d: el caudal medio y la superficie de la cuenca lo están directamente, la ETP y la superficie forestal rala lo están inversamente.

La ETP potencial es un valor que se calcula a partir de la temperatura media y de la latitud; la interpretación de su influencia sobre los caudales no esta clara, sobre todo si no se interpreta junto a la cobertura de la vegetación, pero si parece lógico que una mayor evaporación produzca menos aportes al río. En cuanto a la vegetación, en algunas regiones españolas se ha encontrado que la vegetación influye en que llegue un caudal menor a los ríos en el estiaje (Piñol, 1990).

Estos ríos pertenecen a zonas geológicas similares; todos tienen parte de sus cuencas en el piso bioclimático supramediterraneo, aunque el Guadamejud y Trabaque tienen parte en pisos más bajos y el Tajo en el superior; otras característica similares a todos ellos son sus bajos índice de escorrentía y de erosión pluvial.

Considerando el conjunto de todos los resultados, podemos apreciar que las relaciones obtenidas entre el caudal Q25d y otras variables son similares a las obtenidas por otros trabajos, en los que se relacionan caudales recomendados y variables de cuenca; O’Shea (1995) encuentra que son también el caudal modular y la superficie de la cuenca las variables que más influyen en el caudal mínimo recomendado que él obtie- 
ne, aunque la metodología para calcularlo sea distinta, ya que el usa el método del perímetro mojado (Nelson 1989).

Sería muy interesante determinar las relaciones que existen entre los caudales obtenidos por la metodología IFIM, y los obtenidos con el procedimiento de medias móviles, pues podríamos obtener una comparación de resultados, en los que, posiblemente, se encontraría alguna forma de calcular caudales, con menor trabajo de campo.

Otra razón por la que puede ser también interesante encontrar esta relación entre los dos métodos, es la posibilidad de realizar simulación que tiene la metodología IFIM, puesto que en el empleado en este trabajo sólo se obtienen un caudal, y no se pueden obtener otros valores que se usen para determinar las variaciones estacionales de caudal.

En cuanto a los valores y relaciones obtenidos con el estudio de las cuencas, se ha tratado de completar la información existente entre los aportes de agua a los ríos y las peculiares formas del régimen de estos, consecuencia de cómo les llega esa agua, y las variables que los controlan, entre ellas: el régimen de lluvias, el tipo de acuífero, la altitud, la forma de infiltración junto a los condicionantes que la modifican como los usos del suelo, vegetación, superficie agrícola, granulometría, estructura del suelo, así como las salidas de agua de la cuenca en forma de evaporación.

En este trabajo se ha pensado revisar algunos valores tradicionalmente utilizados en la hidrología torrencial, en los que se incluyen cálculos teóricos y mediciones de estas variables, y comprobar la validez en el estudio de la hidrología de los periodos secos, aunque de momento los datos obtenidos tengan sus limitaciones así como los modelos encontrados.

Algunas variables de la cuenca de las estudiadas han parecido influir en la forma en la que oscilan los caudales en el río, pero hay una clara sectorización y no son las mismas en los grupos de ríos. El comportamiento de cada río es propio, aunque se encuentran razones para estos comportamientos similares en varios ríos y este podría ser un comienzo para un trabajo de sectorización de los ríos de la península, atendiendo por ejem- plo a las variables de cuenca y a las características de sus regímenes.

Las relaciones encontradas en este trabajo pueden ser validadas en otras cuencas, y realizar un estudio más amplio, en el que se estudien estas junto a las características físicas del cauce que se consideran en el método IFIM u otros métodos, de los que ya se tiene alguna información en nuestros ríos, lo que contribuiría a añadir nuevos valores a una mayor caracterización de los ríos y a su posterior clasificación.

\section{BIBLIOGRAFÍA}

BAEZA, D. \& D. GARCÍA DE JALÓN. 1997 Caracterización del régimen de caudales en ríos de la Cuenca del Tajo atendiendo a criterios biológicos. Limnetica, 13: 69-78.

BOVEE, K.D. 1982, A guide to stream habitat analysis using the Instream Flow Incremental Methodology. Instr. Flow Inf. Paper 12. USDI Fish and Wildl. Serv.. Washington. 248 pp.

CEDEX. 1994. Aforos de la cuenca del Tajo. Centro de Estudios y Experimentación de Obras Publicas. Madrid.

CEDEX. 1998. Metodología de cálculo de regímenes de caudales de mantenimiento. Centro de estudios de Técnicas Aplicadas. Madrid.

CUBILLO, F., C. CASADO \& V. CASTILLO. 1990. Estudio de regímenes de caudales mínimos en los cauces de la Comunidad de Madrid. Agencia del Medio Ambiente. Madrid. 305 pp.

GARCÍA DE JALON, D. 1990. Técnicas hidrobiológicas para la fijación de caudales ecológicos mínimos. En: Libro homenaje al profesor D.M. García de Viedma. A. Ramos, A. Notario \& J.R. Baragaño (eds.): 183-196. FUCOVASA.

GUSTARD, A., A. BULLOCK \& J.M. DIXON. 1992. Low flow estimation in the United Kingdom. Report $\mathrm{n}^{\mathrm{o}}$ 108. Institute of Hydrology. Wallingford, UK.

HILL, M. O. 1979. TwINSPAN. a FoRTRAN program for arranging multivariate data in an ordered two-way table by classification of the individuals and attributes. Ecology and Systematics, Cornell University, Ithaca, New York. 90 pp.

MAPA DE APROVECHAMIENTOS Y CULTIVOS. 1977. Ministerio de Agricultura, Pesca y Alimentación. Dirección Provincial de la Producción Agraria. Madrid. 
MAPA DE ESTADOS EROSIVOS DE LA CUENCA DEL TAJO. 1977 Ministerio de Agricultura, Pesca y Alimentación. Madrid.

MAPA GEOTECTÓNICO GENERAL. 1976. Ministerio de Industria. Dirección General de Minas. Instituto Geológico y Minero de España. Madrid.

MAYO, M., A. MINGO \& P.A. BROTONS. 1993. Fijación de regímenes de caudales ecológicos mínimos para los ríos Esla, Porma y Orbigo. Estudio técnico de EAFOR S.A. para AQUATEC S.A. Confederación hidrográfica del Duero. $98 \mathrm{pp}$.

MC MAHON, T.A. 1982. Hydrological characteristics of selected rivers of the world. Unesco pars.

MORA-ALONSO-MUÑOYERRO, J. 1995. Criteria for the determinacion of a regimen of minimum streamflows in regulated rivers- The Spanish situation. In: Reservoirs in Rivers Basin Development. Stanbergen \& Van Western (eds.): 321-331. Balkema, Rotterdam.

MORISAWA, M., 1968. Streams: their dynamics and morphology. New York: McGraw-Hill.

MORISAWA, M.E. 1962. Quantitative geomorphology of some watershead in the Appalachian Plateau. Geological Society of America Bulletin, 73: 1025-1046.

NELSON, P.C.1984. Suitability index (SI) curves for use in the Instream Flow Incremental Methodology: a handy pocket guide. Western Energy and Land Use Team, Instream Flow and Aquatic System Group. Fort Collins, CO.

NELSON, R. L., W.S. PLATTS, D.P. LARSEN \& S.E. JENSEN. 1992 Trout distribution and habitat in relation to geology and geomorphology in the North Fork Humboldt River drainage, Northeastern Nevada. Transactions of the American Fisheries Society, 121: 405-425.

O'SHEA, D T. 1995. Estimating minimum instream flow requirement for Minnesota streams from hydrologic data and watershead characteristics. North American Journal of Fisheries Management, 15: 569-578.

PALAU, A. 1994. Los mal llamados caudales ecológicos. Obras Publicas. Ríos II. N 28.

PALAU, A. \& J. ALCAZAR. 1996. The Basic Flow: An alternative approach to calculate minimum enviromental instream flows. Proc. $2^{\text {nd }}$ International Symposium on Habitat Hydraulics. Ecohydraulics 2000, Quebec. Vol A: 547-558.

PIÑOL, J. 1990 Hidrogeología i biogeoquímica de conques forestades de les muntanyes de Prades. Tesis doctoral, Universidad de Barcelona.

RICHTER, B.D., J. BAUMGARTNER, R. WIGINGTON \& P. BRAUN. 1997. How much water does a river need? Freshwater Biology, 37: 231-249.

SEGUNDO INVENTARIO FORESTAL NACIONAL. 1986-95. Ministerio de Agricultura Pesca y Alimentación. ICONA. Madrid.

STALNAKER, C.B. 1979. The use of habitat structure preference for establishing flow regimes necessary for maintenance of fish habitat. In: The Ecology of Regulated Rivers. J. V. Ward \& J Stanford (eds.): 326-337. Plenum Press, New York. TENNANT, D. L. 1976. Instream Flow Regimens for Fish, Wildlife, Recreation and related Enviromental Resources. Proc. of Instream flow needs Symp.: 326-327.

U.S.D.A. SOIL CONSERVATION SERVICE. 1978. Predicting rainfall erosion losses. - A guide to conservation planning. Agriculture Handbook, 537. Washington.

VALDES. J. B., Y. FIALLO, \& I. RODRIGUEZ ITURBE. 1979. A rainfall-runoff analysis of the geomorphic IUH. Water Resources Research, 15: 1421-1434.

WHITE, R.G. 1976. A methodology for recommending stream resource maintenance flows for large rivers. Proc. of Instream flow needs Symp.: 376-386. 